\title{
On the complete-spectrum characterization of quantum integrable spin chains via inhomogeneous $T-Q$ relation
}

\author{
Junpeng Cao ${ }^{1,2}$, Wen-Li Yang ${ }^{3,4}$, Kangjie Shi ${ }^{3}$ and Yupeng \\ Wang $^{1,2}$ \\ ${ }^{1}$ Beijing National Laboratory for Condensed Matter Physics, Institute of Physics, \\ Chinese Academy of Sciences, Beijing 100190, China \\ ${ }^{2}$ Collaborative Innovation Center of Quantum Matter, Beijing, China \\ ${ }^{3}$ Institute of Modern Physics, Northwest University, Xian 710069, China \\ ${ }^{4}$ Beijing Center for Mathematics and Information Interdisciplinary Sciences, Beijing, \\ 100048, China \\ E-mail: wlyang@nwu.edu.cn (Wen-Li Yang); yupeng@iphy.ac.cn(Yupeng Wang)
}

July 2015

\begin{abstract}
With the XXZ spin chains as examples, we prove two theorems: (1) the functional relations derived from the off-diagonal Bethe Ansatz scheme are the sufficient and necessary conditions to characterize the complete spectrum of the corresponding transfer matrix; (2) each eigenvalue of the transfer matrix can be parameterized by a minimal inhomogeneous $T-Q$ relation. These statements hold for both with and without inhomogeneity. The proof can be generalized to other finite-dimensional quantum integrable models.
\end{abstract}

\section{Introduction}

Recently, a method for solving the eigenvalue problem of quantum integrable models with generic integrable boundary conditions, i.e., the off-diagonal Bethe Ansatz (ODBA) was proposed in [1, 2, 3, 4] and several long-standing models [1, 2, 3, 4, 5, 6, 7, 8, 9, 10] have since been solved (for details, see [11]). The central idea of the method is to construct a proper $T-Q$ relation [12] with an extra off-diagonal (or inhomogeneous) term based on the functional relations among eigenvalues of transfer matrices. However, there are still some concerns about the completeness and uniqueness of the solutions in this scheme [13, 14, 15]. In fact, completeness of Bethe Ansatz solutions or Bethe Ansatz equations (BAEs) for integrable models has been a longstanding problem for many years and some attention has still been paid very recently [16, 17]. In this paper, we take the XXZ spin chains (especially the $U(1)$-symmetry-broken ones) as examples to prove that the functional relations (necessary conditions that the eigenvalues of the transfer matrix obey) derived in the framework of ODBA are also sufficient conditions to 
determine the eigenvalues of the transfer matrix. Therefore, those functional relations completely characterize the spectrum of the transfer matrix in terms of certain minimal inhomogeneous $T-Q$ relation, which gives rise to the Bethe Ansatz solution to the underlying model.

The outline of the paper is as follows. In the next section, after briefly reviewing the construction of the transfer matrices of the XXZ spin- $\frac{1}{2}$ chain with generic open boundary conditions, we give the direct proof that the solution set of the functional relations obtained via ODBA coincides exactly with the set of the eigenvalues of the corresponding transfer matrix. In section 3, we demonstrate that each eigenvalue of the transfer matrix can be parameterized by a polynomial $Q$-function in term of an inhomogeneous $T-Q$ relation. Section 4 is attributed to the case of open spin- $s$ chain. Concluding remarks and some discussion are given in section 5 .

\section{Complete spectrum of the transfer matrix}

The XXZ spin- $\frac{1}{2}$ chain with arbitrary boundary fields is described by the Hamiltonian

$$
\begin{aligned}
H & =\sum_{j=1}^{N-1}\left[\sigma_{j}^{x} \sigma_{j+1}^{x}+\sigma_{j}^{y} \sigma_{j+1}^{y}+\cosh \eta \sigma_{j}^{z} \sigma_{j+1}^{z}\right] \\
& +\frac{\sinh \eta}{\sinh \alpha_{-} \cosh \beta_{-}}\left(\cosh \alpha_{-} \sinh \beta_{-} \sigma_{1}^{z}+\cosh \theta_{-} \sigma_{1}^{x}+i \sinh \theta_{-} \sigma_{1}^{y}\right) \\
& +\frac{\sinh \eta}{\sinh \alpha_{+} \cosh \beta_{+}}\left(-\cosh \alpha_{+} \sinh \beta_{+} \sigma_{N}^{z}+\cosh \theta_{+} \sigma_{N}^{x}+i \sinh \theta_{+} \sigma_{N}^{y}\right),
\end{aligned}
$$

where 6 boundary parameters $\alpha_{ \pm}, \beta_{ \pm}$and $\theta_{ \pm}$are related to the boundary fields. The well-known six-vertex $R$-matrix $R(u) \in \operatorname{End}(V \otimes V)$ (with $V$ a two-dimensional vector space) reads

$$
R(u)=\frac{1}{\sinh \eta}\left(\begin{array}{cccc}
\sinh (u+\eta) & 0 & 0 & 0 \\
0 & \sinh u & \sinh \eta & 0 \\
0 & \sinh \eta & \sinh u & 0 \\
0 & 0 & 0 & \sinh (u+\eta)
\end{array}\right) .
$$

Here and below we adopt the standard notations: for any matrix $A \in \operatorname{End}(\mathrm{V}), A_{j}$ is an embedding operator in the tensor space $V \otimes V \otimes \cdots$, which acts as $A$ on the $j$-th space and as identity on the other factor spaces; $R_{i j}(u)$ is an embedding operator of $R$-matrix in the tensor space, which acts as identity on the factor spaces except for the $i$-th and $j$-th ones. The corresponding transfer matrix is given by [18]

$$
t(u)=\operatorname{tr}_{0}\left\{K_{0}^{+}(u) T_{0}(u) K_{0}^{-}(u) \hat{T}_{0}(u)\right\},
$$

where the one-row monodronomy matrices are given by

$$
\begin{aligned}
& T_{0}(u)=R_{N 0}\left(u-\theta_{N}\right) \ldots R_{10}\left(u-\theta_{1}\right), \\
& \hat{T}_{0}(u)=R_{01}\left(u+\theta_{1}\right) \ldots R_{0 N}\left(u+\theta_{N}\right),
\end{aligned}
$$


$\left\{\theta_{j} \mid j=1, \cdots, N\right\}$ are the generic inhomogeneity parameters, and the $K$-matrices are given by [19, 20]

$$
\begin{aligned}
& K^{-}(u)=\left(\begin{array}{cc}
K_{11}^{-}(u) & K_{12}^{-}(u) \\
K_{21}^{-}(u) & K_{22}^{-}(u)
\end{array}\right), \\
& K_{11}^{-}(u)=2\left(\sinh \left(\alpha_{-}\right) \cosh \left(\beta_{-}\right) \cosh (u)+\cosh \left(\alpha_{-}\right) \sinh \left(\beta_{-}\right) \sinh (u)\right), \\
& K_{22}^{-}(u)=2\left(\sinh \left(\alpha_{-}\right) \cosh \left(\beta_{-}\right) \cosh (u)-\cosh \left(\alpha_{-}\right) \sinh \left(\beta_{-}\right) \sinh (u)\right), \\
& K_{12}^{-}(u)=e^{\theta_{-}} \sinh (2 u), \quad K_{21}^{-}(u)=e^{-\theta_{-}} \sinh (2 u),
\end{aligned}
$$

and

$$
K^{+}(u)=\left.K^{-}(-u-\eta)\right|_{\left(\alpha_{-}, \beta_{-}, \theta_{-}\right) \rightarrow\left(-\alpha_{+},-\beta_{+}, \theta_{+}\right)} .
$$

The commuting property $[t(u), t(v)]=0$ ensures the integrability of the model.

It was shown in [4] that for generic $\left\{\theta_{j} \mid j=1, \ldots, N\right\}$ the transfer matrix given by (2.3) for arbitrary boundary parameters satisfies the properties: $\ddagger$

$$
\begin{aligned}
& t\left(\theta_{j}\right) t\left(\theta_{j}-\eta\right)=a\left(\theta_{j}\right) d\left(\theta_{j}-\eta\right) \times \mathrm{id}, \\
& t(-u-\eta)=t(u), \quad t(u+i \pi)=t(u), \\
& t(0)=-2^{3} \sinh \alpha_{-} \cosh \beta_{-} \sinh \alpha_{+} \cosh \beta_{+} \cosh \eta \\
& \quad \times \prod_{l=1}^{N} \frac{\sinh \left(\eta-\theta_{l}\right) \sinh \left(\eta+\theta_{l}\right)}{\sinh ^{2} \eta} \times \mathrm{id}, \\
& t\left(\frac{i \pi}{2}\right)=-2^{3} \cosh \alpha_{-} \sinh \beta_{-} \cosh \alpha_{+} \sinh \beta_{+} \cosh \eta \\
& \quad \times \prod_{l=1}^{N} \frac{\sinh \left(\frac{i \pi}{2}+\theta_{l}+\eta\right) \sinh \left(\frac{i \pi}{2}+\theta_{l}-\eta\right)}{\sinh ^{2} \eta} \times \mathrm{id}, \\
& \lim _{u \rightarrow \pm \infty} t(u)=-\frac{\cosh \left(\theta_{-}-\theta_{+}\right) e^{ \pm[(2 N+4) u+(N+2) \eta]}}{2^{2 N+1} \sinh ^{2 N} \eta} \times \mathrm{id}+\ldots,
\end{aligned}
$$

where the functions $a(u)$ and $d(u)$ are given by [4]

$$
\begin{aligned}
a(u)= & -2 \frac{\sinh (2 u+2 \eta)}{\sinh (2 u+\eta)} \sinh \left(u-\alpha_{-}\right) \\
& \times \cosh \left(u-\beta_{-}\right) \sinh \left(u-\alpha_{+}\right) \cosh \left(u-\beta_{+}\right) A(u), \\
d(u)= & a(-u-\eta), \quad A(u)=\prod_{l=1}^{N} \frac{\sinh \left(u-\theta_{l}+\eta\right) \sinh \left(u+\theta_{l}+\eta\right)}{\sinh ^{2}(\eta)} .
\end{aligned}
$$

The above operator relations lead to that the corresponding eigenvalue, denoted by $\Lambda(u)$, of the transfer matrix enjoys the properties:

$$
\Lambda\left(\theta_{j}\right) \Lambda\left(\theta_{j}-\eta\right)=a\left(\theta_{j}\right) d\left(\theta_{j}-\eta\right), \quad j=1, \ldots, N,
$$

$\ddagger$ The relations (see (2.14) below) satisfied by the eigenvalues of the transfer matrix for the XXZ spin$\frac{1}{2}$ open chain with one general non-diagonal and one diagonal or triangular boundary K-matrices was previously obtained by the separation of variables method [21. The first proof of the relation (2.7) for arbitrary boundary parameters on the operator level (which does not depend on the basis) was given in the reference [4]. 


$$
\begin{gathered}
\Lambda(-u-\eta)=\Lambda(u), \quad \Lambda(u+i \pi)=\Lambda(u) \\
\Lambda(0)=-2^{3} \sinh \alpha_{-} \cosh \beta_{-} \sinh \alpha_{+} \cosh \beta_{+} \cosh \eta \\
\quad \times \prod_{l=1}^{N} \frac{\sinh \left(\eta-\theta_{l}\right) \sinh \left(\eta+\theta_{l}\right)}{\sinh ^{2} \eta} \\
\Lambda\left(\frac{i \pi}{2}\right)=-2^{3} \cosh \alpha_{-} \sinh \beta_{-} \cosh \alpha_{+} \sinh \beta_{+} \cosh \eta \\
\quad \times \prod_{l=1}^{N} \frac{\sinh \left(\frac{i \pi}{2}+\theta_{l}+\eta\right) \sinh \left(\frac{i \pi}{2}+\theta_{l}-\eta\right)}{\sinh ^{2} \eta}, \\
\lim _{u \rightarrow \pm \infty} \Lambda(u)=-\frac{\cosh \left(\theta_{-}-\theta_{+}\right) e^{ \pm[(2 N+4) u+(N+2) \eta]}}{2^{2 N+1} \sinh ^{2 N} \eta}+\ldots,
\end{gathered}
$$

$\Lambda(u)$, as an entire function of $u$,

$$
\text { is a trigonometric polynomial of degree } 2 N+4 \text {. }
$$

The analogue of the above relations in the homogeneous limit reads:

$$
\begin{aligned}
& \left.\frac{\partial^{l}}{\partial u^{l}} \Lambda(u) \Lambda(u-\eta)\right|_{u=0} \\
& =\left.\frac{\partial^{l}}{\partial u^{l}}\left\{\left.\left.a(u)\right|_{\theta_{j}=0} d(u)\right|_{\theta_{j}=0}\right\}\right|_{u=0}, \quad l=0,1, \ldots, N-1 \\
& \Lambda(-u-\eta)=\Lambda(u), \quad \Lambda(u+i \pi)=\Lambda(u) \\
& \Lambda(0)=-2^{3} \sinh \alpha_{-} \cosh \beta_{-} \sinh \alpha_{+} \cosh \beta_{+} \cosh \eta \\
& \Lambda\left(\frac{i \pi}{2}\right)=(-1)^{N+1} 2^{3} \cosh \alpha_{-} \sinh \beta_{-} \cosh \alpha_{+} \sinh \beta_{+} \cosh \eta \frac{\cosh ^{2 N} \eta}{\sinh ^{2 N} \eta} \\
& \lim _{u \rightarrow \pm \infty} \Lambda(u)=-\frac{\cosh \left(\theta_{-}-\theta_{+}\right) e^{ \pm[(2 N+4) u+(N+2) \eta]}}{2^{2 N+1} \sinh ^{2 N} \eta}+\ldots \\
& \Lambda(u), \text { as an entire function of } u, \\
& \quad \text { is a trigonometric polynomial of degree } 2 N+4
\end{aligned}
$$

Proposition 1 The relations (2.14)-(2.19) completely characterize the spectrum of the transfer matrix given by (2.3) for the inhomogeneous XXZ spin- $\frac{1}{2}$ open chain with the most generic non-diagonal $K$-matrices specified by (2.5) and (2.6).

Proof. An important fact is that for generic $\eta$ the generic boundary fields break all the non-abelian symmetries of the Hamiltonian and induce non-degeneracy of the spectrum. Since all the eigenvalues of the transfer matrix must belong to the solutions of (2.14)(2.19), we conclude that the number of unequal solutions of (2.14)-(2.19) must be larger or equal to $2^{N}$ (dimension of the Hilbert space)\&s. On the other hand, the equations (2.15) and (2.18)-(2.19) imply that the solutions form a $N+3$-dimensional linear space and each of the solutions can be expressed uniquely in terms of $N+3$ unknown coefficients $\S$ Note that this conclusion does not hold if there is some degeneracy in the spectrum. 
$\left\{\bar{I}_{i} \mid i=0, \ldots, N+2\right\}$ as follows $Џ$

$$
\Lambda(u)=\sum_{i=0}^{N+2} \bar{I}_{i}\left(e^{(2 N+4-2 i) u}+e^{-(2 N+4-2 i)(u+\eta)}\right) .
$$

Substituting the above expression into (2.14) and (2.16) $-(2.18)$ gives rise to $N+3$ equations with respect to the $N+3$ unknown coefficients $\left\{\bar{I}_{i} \mid i=0, \ldots, N+2\right\}$. Among the resulting equations, $N$ of them are quadratic in terms of $\left\{\bar{I}_{i} \mid i=0, \ldots, N+2\right\}$ and the other 3 equations are linear in terms of $\left\{\bar{I}_{i} \mid i=0, \ldots, N+2\right\}$. According to the Bézout Theorem [22] is $2^{N} \times 1^{3}=2^{N}$. Based on the above arguments we conclude that the number of solutions is exactly $2^{N}$ and the solution set of $(\underline{2.14})-(2.19)$ is exactly the eigenvalue set of the transfer matrix.

Similarly, we have

Corollary 1 For the homogenous XXZ spin- $\frac{1}{2}$ open chain with the most generic nondiagonal $K$-matrices specified by (2.5) and (2.6), the spectrum of the transfer matrix is completely determined by the equations (2.20)-(2.25).

It was shown [21] that for each solution to (2.14)-(2.19), only with non-trivial inhomogeneity, one could construct the corresponding eigenstate (i.e., the SoV-type eigensate whose homogeneous limit is still unclear) of the transfer matrix. However, the corresponding Bethe-type eigensate, no matter with or without inhomogeneity, can be constructed [23, 24, 25] associated with each solution to (2.14) $-(2.19)($ or $(2.20)-(2.25))+$

\section{Inhomogeneous $T-Q$ relation}

In this section, we show that each eigenvalue of the transfer matrix (the solution of $(\underline{2.14})-(2.19))$ can be expressed in terms of some inhomogeneous $T-Q$ relation proposed in [4].

Proposition 2 Each solution of (2.14)-(2.19) can be parameterized in terms of the inhomogeneous $T-Q$ relation

$$
\begin{aligned}
\Lambda(u) Q(u)= & a(u) Q(u-\eta)+d(u) Q(u+\eta) \\
& +2 c \sinh 2 u \sinh (2 u+2 \eta) A(u) A(-u-\eta),
\end{aligned}
$$

with $Q(u)$ being a trigonometric polynomial as

$$
Q(u)=\prod_{j=1}^{N} \frac{\sinh \left(u-\lambda_{j}\right) \sinh \left(u+\lambda_{j}+\eta\right)}{\sinh ^{2} \eta},
$$

\| One may adopt another basis $\left\{(\sinh u \sinh (u+\eta))^{i}(\cosh u \cosh (u+\eta))^{N+2-i} \mid i=0, \ldots, N+2\right\}$.

I The Bézout Theorem states that given two algebraic curves $\mathrm{X}$ and $\mathrm{Y}$, the maximum number of their common points is the product of their degrees.

+ It should be emphasized that the inhomogeneous $T-Q$ relation formalism (see (3.1) below) plays a key role in constructing the Bethe-type eigenstates [23, 24, 25]. 
and the constant $c$ being given by

$$
c=\cosh \left(\alpha_{-}+\beta_{-}+\alpha_{+}+\beta_{+}+(1+N) \eta\right)-\cosh \left(\theta_{-}-\theta_{+}\right) .
$$

The $N$ parameters $\left\{\lambda_{j}\right\}$ satisfy the associated BAEs

$$
\begin{gathered}
a\left(\lambda_{j}\right) Q\left(\lambda_{j}-\eta\right)+d\left(\lambda_{j}\right) Q\left(\lambda_{j}+\eta\right)+2 c \sin 2 \lambda_{j} \sinh \left(2 \lambda_{j}+2 \eta\right) \\
\times A\left(\lambda_{j}\right) A\left(-\lambda_{j}-\eta\right)=0, \quad j=1, \ldots, N .
\end{gathered}
$$

Proof. Let us introduce a function $f(u)$ which is equal to the difference between the LHS and the RHS of (3.1), namely,

$$
\begin{aligned}
f(u)= & \Lambda(u) Q(u)-a(u) Q(u-\eta) \\
& -d(u) Q(u+\eta)-2 c \sinh 2 u \sinh (2 u+2 \eta) A(u) A(-u-\eta) .
\end{aligned}
$$

The relations (2.15) and (2.19) allow us to derive that $f(u)$ satisfies the properties:

$$
\begin{aligned}
& f(u+i \pi)=f(u), \\
& f(u), \text { as a function of } u, \\
& \quad \text { is a trigonometrical polynomial of degree } 4 N+4 \text {. }
\end{aligned}
$$

This implies that together with the crossing symmetry, the function $f(u)$ is fixed by its values at any $2 N+3$ different points. For each solution of (2.14) $-(2.19)$, one can always choose a $Q(u)$ of form (3.2) such that

$$
\begin{aligned}
& f(0)=f\left(\frac{i \pi}{2}\right)=f(\infty)=0 \\
& f\left(\theta_{j}\right)=\Lambda\left(\theta_{j}\right) Q\left(\theta_{j}\right)-a\left(\theta_{j}\right) Q\left(\theta_{j}-\eta\right)=0, \quad j=1, \ldots, N \\
& f\left(\theta_{j}-\eta\right)=\Lambda\left(\theta_{j}-\eta\right) Q\left(\theta_{j}-\eta\right)-d\left(\theta_{j}-\eta\right) Q\left(\theta_{j}\right)=0, j=1, \ldots, N,
\end{aligned}
$$

which means $f(u)=0$ or (3.1) is fulfilled. In fact, the relation (3.8) is automatically satisfied, while the remaining $2 N$ equations (3.9)-(3.10) can be rewritten in terms of the following $N$ two-components equations

$$
\left(\begin{array}{cc}
\Lambda\left(\theta_{j}\right) & -a\left(\theta_{j}\right) \\
d\left(\theta_{j}-\eta\right) & \Lambda\left(\theta_{j}-\eta\right)
\end{array}\right)\left(\begin{array}{c}
Q\left(\theta_{j}\right) \\
Q\left(\theta_{j}-\eta\right)
\end{array}\right)=\left(\begin{array}{l}
0 \\
0
\end{array}\right), j=1, \ldots, N
$$

The conditions that the above $N$ equations have non-trivial solutions is exact (2.14). This means that if $\Lambda(u)$ is a solution of (2.14) $-(2.19)$ ), the $2 N$ equations (3.9) and (3.10) are equivalent to $N$ equations

$$
\Lambda\left(\theta_{j}\right) Q\left(\theta_{j}\right)=a\left(\theta_{j}\right) Q\left(\theta_{j}-\eta\right), \quad j=1, \ldots, N,
$$

which allow us to determine the $Q(u)$ function in the form of (3.2) by its values at the $N$ points $\theta_{j}$. Therefore, we are always able to choose the $Q(u)$ of form (3.2) from (3.12) such that $f(u)=0$ provided that $\Lambda(u)$ is an eigenvalue of the transfer matrix (2.3). Moreover, taking $u$ at the roots of the $Q(u)$ function (i.e., $\left.\left\{\lambda_{j}\right\}\right)$, the condition 
$f\left(\lambda_{j}\right)=0$ gives rise to the associated BAEs (3.4), which determine the $Q(u)$ function * This completes the proof.

Some remarks are in order. (1) Provided that all the eigenvalues $\Lambda(u)$ are simple, $N$ is the minimal degree of the trigonometric polynomial $Q(u)$ (3.2) enabling one to parameterize any eigenvalue $\Lambda(u)$ in the form of (3.1). (2) Actually there exist an infinite number of possible ways [4] to parameterize the eigenvalue of the transfer matrix, but they are all equivalent to each other because of the finite number of eigenvalues. The corresponding Bethe states for different parametrization were constructed [23, 24, 25]. (3) The degree of the $Q$-polynomial may be reduced to a small value for the case that the inhomogeneous term vanishes. In this case the $T-Q$ relation becomes a homogeneous one (the well-known Baxter's $T-Q$ relation). This happens in cases of the $U(1)$ symmetry or in the degenerate cases of the open spin chain [26], where the transfer matrix can be diagonalized in some smaller blocks.

\section{Results for the XXZ spin- $s$ open chain}

For the XXZ spin- $s$ chain [27, 28, 29, 30, 31, 32] $\left(s=\frac{1}{2}, 1, \frac{3}{2}, \ldots\right)$, the quantum space of each site is $2 s+1$-dimensional and endows the spin- $s$ representation of $U_{q}\left(s l_{2}\right)$ with $q=e^{\eta}$ [33]. The fundamental spin- $\left(\frac{1}{2}, s\right) R$-matrix is given by [28, 29, 30, 31]

$$
R_{12}^{\left(\frac{1}{2}, s\right)}(u)=\frac{1}{\sinh \eta}\left(\begin{array}{cc}
\sinh \left(u+\frac{\eta}{2}+\eta S_{2}^{3}\right) & \sinh \eta S_{2}^{-} \\
\sinh \eta S_{2}^{+} & \sinh \left(u+\frac{\eta}{2}-\eta S_{2}^{3}\right)
\end{array}\right),
$$

where $S^{3}, S^{ \pm}$are the spin-s realizations of the quantum algebra $U_{q}\left(s l_{2}\right)$. The fundamental transfer matrix denoted by $t^{\left(\frac{1}{2}, s\right)}(u)$, is given by (cf. (2.3) )

$$
t^{\left(\frac{1}{2}, s\right)}(u)=\operatorname{tr}_{0}\left\{K_{0}^{+}(u) T_{0}^{\left(\frac{1}{2}, s\right)}(u) K_{0}^{-}(u) \hat{T}_{0}^{\left(\frac{1}{2}, s\right)}(u)\right\},
$$

where the two monodromy matrices are given by

$$
\begin{aligned}
& T^{\left(\frac{1}{2}, s\right)}(u)=R_{0 N}^{\left(\frac{1}{2}, s\right)}\left(u-\theta_{N}\right) \ldots R_{01}^{\left(\frac{1}{2}, s\right)}\left(u-\theta_{1}\right), \\
& \hat{T}^{\left(\frac{1}{2}, s\right)}(u)=R_{01}^{\left(\frac{1}{2}, s\right)}\left(u+\theta_{1}\right) \ldots R_{0 N}^{\left(\frac{1}{2}, s\right)}\left(u+\theta_{N}\right),
\end{aligned}
$$

and the corresponding $K$-matrices are given by (2.5) and (2.6) respectively. The transfer matrix and its fused ones (for details we refer to [34, 35, 36, 37]), denoted by $t^{(j, s)}(u)$, form the commutative families, which ensures the integrability of the corresponding model. Moreover these satisfy the fusion hierarchy [38] relations [39, 40, 41, 42]

$$
\begin{aligned}
& t^{\left(\frac{1}{2}, s\right)}(u) t^{\left(j-\frac{1}{2}, s\right)}(u-j \eta)=t^{(j, s)}\left(u-\left(j-\frac{1}{2}\right) \eta\right) \\
&+\delta^{(s)}(u) t^{(j-1, s)}\left(u-\left(j+\frac{1}{2}\right) \eta\right), \quad j=\frac{1}{2}, 1, \frac{3}{2}, \ldots,
\end{aligned}
$$

\footnotetext{
* Note that we only claim that each solution of $\Lambda(u)$ can be parameterized by an inhomogeneous $T-Q$ relation. This does not mean that all $T-Q$ relations given by the solutions of the BAEs correspond to right eigenvalues because there may be "unphysical" solutions to the BAEs as discussed in [16, 17. Nevertheless we would point out that the "irregular" solutions of the BAEs with $\lambda_{j}=\theta_{j}, \theta_{j}-\eta$ do not satisfy (2.14) in our case.
} 
where we have used the convention $t^{(0, s)}=\mathrm{id}$ and the coefficient function $\delta^{(s)}(u)$ related to the quantum determinant is given by

$$
\begin{aligned}
\delta^{(s)}(u)= & \bar{a}^{(s)}(u) d^{(s)}(u-\eta), \\
\bar{a}^{(s)}(u)= & -2^{2} \frac{\sinh (2 u+2 \eta)}{\sinh (2 u+\eta)} \sinh \left(u-\alpha_{-}\right) \cosh \left(u-\beta_{-}\right) \\
& \times \sinh \left(u-\alpha_{+}\right) \cosh \left(u-\beta_{+}\right) \bar{A}^{(s)}(u), \\
\bar{d}^{(s)}(u)= & \bar{a}^{(s)}(-u-\eta), \\
\bar{A}^{(s)}(u)= & \prod_{l=1}^{N} \frac{\sinh \left(u-\theta_{l}+\left(\frac{1}{2}+s\right) \eta\right) \sinh \left(u+\theta_{l}+\left(\frac{1}{2}+s\right) \eta\right)}{\sinh ^{2}(\eta)} .
\end{aligned}
$$

The fused transfer matrices $t^{(j, s)}(u)$, which are mutually commuting, can be constructed by the fused $R$-matrices and $K$-matrices [28, 32, 39, 40, 42]. Let us denote the eigenvalue of the transfer matrix $t^{(j, s)}(u)$ by $\Lambda^{(j, s)}(u)$. The commutativity of the transfer matrices and the operator relations (4.2) imply that the eigenvalues also obey the same fusion hierarchy relations

$$
\begin{aligned}
\Lambda^{\left(\frac{1}{2}, s\right)}(u) \Lambda^{\left(j-\frac{1}{2}, s\right)}(u-j \eta) & =\Lambda^{(j, s)}\left(u-\left(j-\frac{1}{2}\right) \eta\right) \\
& +\delta^{(s)}(u) \Lambda^{(j-1, s)}\left(u-\left(j+\frac{1}{2}\right) \eta\right), \quad j=\frac{1}{2}, 1, \frac{3}{2}, \ldots
\end{aligned}
$$

Following the method developed in [7, 9], we can obtain some operator identities between $t^{\left(\frac{1}{2}, s\right)}(u)$ and $t^{(s, s)}(u)$ at some special points, which leads to the following relation among their eigenvalues [10, 43]

$$
\begin{aligned}
& \Lambda^{(s, s)}\left(\theta_{j}\right) \Lambda^{\left(\frac{1}{2}, s\right)}\left(\theta_{j}-\left(\frac{1}{2}+s\right) \eta\right) \\
& =\delta^{(s)}\left(\theta_{j}+\left(\frac{1}{2}-s\right) \eta\right) \Lambda^{\left(s-\frac{1}{2}, s\right)}\left(\theta_{j}+\frac{\eta}{2}\right), \quad j=1, \ldots, N .
\end{aligned}
$$

Moreover, it is easy to verify that $\Lambda^{\left(\frac{1}{2}, s\right)}(u)$ satisfy the relations:

$$
\begin{aligned}
& \Lambda^{\left(\frac{1}{2}, s\right)}(u+i \pi)=\Lambda^{\left(\frac{1}{2}, s\right)}(u), \quad \Lambda^{\left(\frac{1}{2}, s\right)}(-u-\eta)=\Lambda^{\left(\frac{1}{2}, s\right)}(u), \\
& \Lambda^{\left(\frac{1}{2}, s\right)}(0)=-2^{3} \sinh \alpha_{-} \cosh \beta_{-} \sinh \alpha_{+} \cosh \beta_{+} \cosh \eta \\
& \times \prod_{l=1}^{N} \sinh \left(\theta_{l}+\left(\frac{1}{2}+s\right) \eta\right) \sinh \left(-\theta_{l}+\left(\frac{1}{2}+s\right) \eta\right), \\
& \Lambda^{\left(\frac{1}{2}, s\right)}\left(\frac{i \pi}{2}\right)=-2^{3} \cosh \alpha_{-} \sinh \beta_{-} \cosh \alpha_{+} \sinh \beta_{+} \cosh \eta \\
& \times \prod_{l=1}^{N} \sinh \left(\frac{i \pi}{2}+\theta_{l}+\left(\frac{1}{2}+s\right) \eta\right) \sinh \left(\frac{i \pi}{2}+\theta_{l}-\left(\frac{1}{2}+s\right) \eta\right), \\
& \left.\Lambda^{\left(\frac{1}{2}, s\right)}(u)\right|_{u \rightarrow \pm \infty}=-\frac{\cosh \left(\theta_{-}-\theta_{+}\right) e^{ \pm[(2 N+4) u+(N+2) \eta]}}{2^{2 N+1} \sinh ^{2 N} \eta}+\ldots, \\
& \Lambda^{\left(\frac{1}{2}, s\right)}(u) \text {, as a function of } u \text {, } \\
& \text { is a trigonometric polynomial of degree } 2 N+4 \text {. }
\end{aligned}
$$


The equations (4.9), (4.12)-(4.13) allow us to express $\Lambda^{\left(\frac{1}{2}, s\right)}(u)$ uniquely in terms of $N+3$ unknown coefficients $\left\{\bar{I}_{i}^{(s)} \mid i=0, \ldots, N+2\right\}$ as follow

$$
\Lambda^{\left(\frac{1}{2}, s\right)}(u)=\sum_{i=0}^{N+2} \bar{I}_{i}^{(s)}\left(e^{(2 N+4-2 i) u}+e^{-(2 N+4-2 i)(u+\eta)}\right) .
$$

The hierarchy relation (4.7) implies that $\Lambda^{(s, s)}(u)$, as a function of $\left\{\bar{I}_{i}^{(s)} \mid i=0, \ldots, N+2\right\}$, is a polynomial of degree $2 s$. Then each equation of (4.8), with respect to $\left\{\bar{I}_{i}^{(s)} \mid i=\right.$ $0, \ldots, N+2\}$, is of degree $2 s+1$. Noting the fact that the dimension of the Hilbert space of the spin- $s$ chain is $(2 s+1)^{N}$, with similar analysis for the spin- $\frac{1}{2}$ case we conclude that the equations (4.7)-(4.13) completely characterize the spectrum of the transfer matrix $t^{\left(\frac{1}{2}, s\right)}(u)$, and that each eigenvalue $\Lambda^{\left(\frac{1}{2}, s\right)}(u)$ of the transfer matrix $t^{\left(\frac{1}{2}, s\right)}(u)$ can be parameterized by the inhomogeneous $T-Q$ relation

$$
\begin{aligned}
\Lambda^{\left(\frac{1}{2}, s\right)}(u)= & a^{(s)}(u) \frac{Q(u-\eta)}{Q(u)}+d^{(s)}(u) \frac{Q(u+\eta)}{Q(u)} \\
& +2 c^{(s)} \sinh 2 u \sinh (2 u+2 \eta) \frac{F^{(s)}(u)}{Q(u)},
\end{aligned}
$$

where the function $F^{(s)}(u)$ and the constant $c^{(s)}$ are given by

$$
\begin{aligned}
& F^{(s)}(u)=\prod_{j=1}^{N} \prod_{k=0}^{2 s} \frac{\sinh \left(u-\theta_{j}+\left(\frac{1}{2}-s+k\right) \eta\right) \sinh \left(u+\theta_{j}+\left(\frac{1}{2}-s+k\right) \eta\right)}{\sinh \eta \sinh \eta} \\
& c^{(s)}=\cosh \left(\alpha_{-}+\beta_{-}+\alpha_{+}+\beta_{+}+(1+2 s N) \eta\right)-\cosh \left(\theta_{-}-\theta_{+}\right)
\end{aligned}
$$

The function $Q(u)$ is parameterized by $2 s N$ Bethe roots $\left\{\lambda_{j} \mid j=1, \ldots, 2 s N\right\}$ as

$$
Q(u)=\prod_{j=1}^{2 s N} \frac{\sinh \left(u-\lambda_{j}\right) \sinh \left(u+\lambda_{j}+\eta\right)}{\sinh \eta \sinh \eta}=Q(-u-\eta),
$$

which should satisfy the BAEs

$$
\begin{aligned}
& a^{(s)}\left(\lambda_{j}\right) Q\left(\lambda_{j}-\eta\right)+d^{(s)}\left(\lambda_{j}\right) Q\left(\lambda_{j}+\eta\right) \\
& \quad+2 c^{(s)} \sinh 2 \lambda_{j} \sinh \left(2 \lambda_{j}+2 \eta\right) F^{(s)}\left(\lambda_{j}\right)=0, \quad j=1, \ldots, 2 s N .
\end{aligned}
$$

\section{Conclusions}

The complete-spectrum characterization of quantum integrable spin chains via ODBA is studied. Taking the XXZ spin chains (especially for the $U(1)$-symmetry-broken ones) as examples, we have shown that the relations (2.14)-(2.19) completely characterize the spectrum of the transfer matrix of the spin- $\frac{1}{2} \mathrm{XXZ}$ chain with generic non-diagonal open boundary condition. It is further shown that each eigenvalue of the transfer matrices can be parameterized in terms of some inhomogeneous $T-Q$ relation such as (3.1), which gives rise to the Bethe Ansatz solution to the underlying model [1]. Moreover, for the spin- $s$ chain, the relations (4.7)-(4.13) completely characterize the spectrum of the fundamental transfer matrix $t^{\left(\frac{1}{2}, s\right)}(u)$ and each eigenvalue can be given in terms of the inhomogeneous $T-Q$ relation (4.14). 
The inhomogeneous $T-Q$ relation of type (3.1) or (4.14), like the role of the YangBaxter equation, plays a unified and fundamental role to characterize the eigenvalue of the transfer matrix of rank-one quantum integrable models both with and without $U(1)$ symmetry.

\section{Acknowledgments}

We would like to thank F. Gong for drawing our attention to the Bézout Theorem. The financial supports from the National Natural Science Foundation of China (Grant Nos. 11375141, 11374334, 11434013 and 11425522), the National Program for Basic Research of MOST (973 project under grant No. 2011CB921700) and BCMIIS are gratefully acknowledged. Two of the authors (W.-L. Yang and K. Shi) would like to thank IoP, CAS for the hospitality. W.-L. Yang also would like to thank KITPC for the hospitality where some part of the work was done during his visiting.

\section{References}

[1] Cao J, Yang W -L, Shi K and Wang Y 2013 Phys. Rev. Lett. 111137201

[2] Cao J, Yang W -L, Shi K and Wang Y 2013 Nucl. Phys. B 875152

[3] Cao J, Cui S, Yang W -L, Shi K and Wang Y 2014 Nucl. Phys. B 886185

[4] Cao J, Yang W -L, Shi K and Wang Y 2013 Nucl. Phys. B 877152

[5] Li Y -Y, Cao J, Yang W -L, Shi K and Wang Y 2014 Nucl. Phys. B 87998

[6] Zhang X, Cao J, Yang W -L, Shi K and Wang Y 2014 J. Stat. Mech. P04031

[7] Cao J, Yang W -L, Shi K and Wang Y 2014 JHEP 04143

[8] Li Y -Y, Cao J, Yang W -L, Shi K and Wang Y 2014 Nucl. Phys. B 88417

[9] Hao K, Cao J, Li G -L, Yang W -L, Shi K and Wang Y 2014 JHEP 06128

[10] Cao J, Cui S, Yang W -L, Shi K and Wang Y 2015 JHEP 02036

[11] Wang Y, Yang W -L, Cao J and Shi K 2015 Off-Diagonal Bethe Ansatz for Exactly Solvable Models (Berlin-Heidelberg: Springer)

[12] Baxter R J 1982 Exactly Solved Models in Statistical Mechanics (London: Academic)

[13] Nepomechie R I 2013 J. Phys. A 46442002

[14] Jiang Y, Cui S, Cao J, Yang W -L and Wang Y 2013 arXiv:1309.6456

[15] Kitanine N, Maillet J -M, Niccoli G 2014 J. Stat. Mech. P05015

[16] Hao W, Nepomechie R I and Sommese A J 2013 Phys. Rev. E 88052113

[17] Hao W, Nepomechie R I and Sommese A J 2013 arXiv:1312.2982

[18] Sklyanin E K 1988 J. Phys. A 212375

[19] de Vega H J and González-Ruiz A 1994 J. Phys. A 276129

[20] Ghoshal S and Zamolodchikov A B 1994 Int. J. Mod. Phys. A 93841

[21] Faldella S, Kitanine N and Niccoli G 2014 J. Stat. Mech. P01011

[22] Griffiths P and Harris J 1994 Principles of Algebraic Geometry (New York: Wiley Classics Library)

[23] Zhang X, Li Y -Y, Cao J, Yang W -L, Shi K and Wang Y 2015 J. Stat. Mech. P05014

[24] Belliard S 2015 Nucl. Phys. B 8921

[25] Zhang X, Li Y -Y, Cao J, Yang W -L, Shi K and Wang Y 2015 Nucl. Phys. B 89370

[26] Cao J, Lin H -Q, Shi K -J and Wang Y 2003 Nucl. Phys. B 663487

[27] Zamolodchikov A B and Fateev V A 1980 Sov. J. Nucl. Phys. 32298

[28] Kulish P P and Sklyanin E K 1982 Lecture Notes in Physics 15161

[29] Kulish P P and Reshetikhin N Yu 1983 J. Sov. Math. 232435

[30] Kirillov A N and Reshetikhin N Yu 1986 J. Sov. Math. 352627 
[31] Kirillov A N and Reshetikhin N Yu 1987 J. Phys. A 201565

[32] Mezincescu L, Nepomechie R I and Rittenberg V 1990 Phys. Lett. A 14770

[33] Chari V and Pressley A 1994 A Guide to Quantum Groups (Cambridge: Cambridge University)

[34] Nepomechie R I 2001 J. Phys. A 349993

[35] Nepomechie R I 2002 Nucl. Phys. B 622615

[36] Nepomechie R I 2003 J. Stat. Phys. 1111363

[37] Nepomechie R I 2004 J. Phys. A 37433

[38] Kulish P P, Reshetikhin N Yu and Sklyanin E K 1981 Lett. Math. Phys. 5393

[39] Mezincescu L and Nepomechie R I 1992 J. Phys. A 252533

[40] Zhou Y -K 1996 Nucl. Phys. B 458504

[41] Yang W -L, Nepomechie R I and Zhang Y -Z 2006 Phys. Lett. B 633664

[42] Frappat L, Nepomechie R I and Ragoncy E 2007 J. Stat. Mech. P09008

[43] Cao J, Yang W -L, Shi K and Wang Y 2015 Ann. Phys. 36191 